CARDIOVASCULAR SURGERY OUTCOMES OF PATIENTS WITH HIV: NEW DATA IN THE ERA OF COMBINATION ANTIRETROVIRAL THERAPY Reply to the Editor:

We thank Sullivan and colleagues for their interest in our article regarding cardiovascular surgical outcomes of patients with human immunodeficiency virus (HIV). The advantage of using a large administrative data set such as the Nationwide Inpatient Sample is the ability to examine a relatively rare clinical situation. In our study, we were able to examine the outcomes of a large number of HIV-seropositive patients undergoing cardiac operations. One significant limitation, however, is the lack of clinical data. Clearly, understanding the status of the patients' disease would be beneficial, especially with regard to $\mathrm{CD}^{+}$ T-cell counts. We agree that the degree to which HIV is controlled or the development of acquired immunodeficiency syndrome would significantly impact the outcome.

The etiology of the heart disease is not easy to discern, and we appreciate that the treatment for HIV has evolved significantly with time. We found it interesting that patients with HIV were seen significantly earlier (49 years old vs 66 years old). It is not entirely clear why this was the case, but it may be due to the HIV or the treatment. It is likely that many of the patients who were operated on during our study period were treated with early-generation antiretroviral regimens, and that may have contributed to cardiac disease progression. It will be interesting to see how the trend in cardiac operations changes in the future in response to the newer protease inhibitors.

The article by Hsu and coworkers cited in the letter of Sullivan and colleagues offers an excellent look at the de novo development of atrial fibrillation and flutter in a large cohort of HIV-seropositive patients. Hsu and coworkers noted that not only did traditional risk factors such as age, race, coronary artery disease, and congestive heart failure play a role, but HIV severity also had a significant impact. Adding the stress and inflammatory response to cardiac surgery and cardiopulmonary bypass further complicates the situation. It is not well understood how the immunodeficient HIV-seropositive patient responds to these stressors. In our article, we comment on the fact that in the article of Hsu and coworkers atrial fibrillation was higher in HIV-seropositive patients, but in our own study arrhythmia was less common in the HIV-seropositive patients (Table 2 in our original article). The fact is, we really do not know why HIV-seropositive patients had a lower incidence of stroke, and a lower rate of postoperative atrial fibrillation was one supposition. It could also be that HIV-seropositive patients are younger and do not have the same burden of aortic atherosclerotic disease.

We read the recent article by Longenecker and colleagues with great interest, as this review sought to clarify the relationship of HIV and the treatment regimens with regard to development of cardiovascular disease. This thoughtful article concludes that treatment of HIV should be started earlier, at higher $\mathrm{CD}^{+}$counts, because uncontrolled viral replication is likely an important contributor to the development of cardiovascular disease, and modern treatments are safer in this regard.

We fully agree that more robust studies of the topic are needed to further our understanding of the interplay between HIV and antiretroviral therapy. We look forward to the results of the Strategic Timing of Anti-Retroviral Treatment (START) trial, which will examine cardiovascular disease as a secondary outcome. In summary, we were encouraged to see that patients with HIV can have excellent outcomes of cardiac operations, and we hope that as more of these patients live longer and healthier lives, we can provide the appropriate care.

Michael Robich, $M D$

Edward Soltesz, MD, MPH

Department of Thoracic and Cardiovascular Surgery Cleveland Clinic Foundation Cleveland, Ohio

http://dx.doi.org/10.1016/ j.jtcvs.2015.02.027

\section{THE CASE FOR MULTIPLE ARTERIAL CORONARY ARTERY BYPASS GRAFT: NO LONGER A LEAP OF FAITH To the Editor:}

We wholeheartedly agree with the assertion of Halkos and Guyton that the time has come for a "palpable shift in our practice of coronary revascularization" away from the traditional left internal thoracic artery (LITA)-based single arterial coronary artery bypass graft (SABG) to either radial artery (RA) or right internal thoracic artery (RITA)-based multiarterial coronary artery bypass graft (MABG). ${ }^{1}$ We applaud and fully support the authors' impassioned plea for the adoption of MABG into routine everyday practice. Although their reservations regarding $\mathrm{MABG}$ are well articulated, they may be overstated and do not fully convey the current evidence supporting MABG. We contend that, in the absence of any meaningful data substantiating a survival disadvantage associated with MABG compared with SABG, the evidence in favor of MABG as the superior technique can no longer be ignored, and that waiting for the complete and unambiguous resolution of these reservations through randomized trials before the wider adoption of MABG represents a disservice to our patients. In the face of the current MABG utilization rate of $9 \%$ among the Society of 